\title{
Association between dissociated firing in isolated pulmonary veins and the initiation and maintenance of atrial fibrillation
}

\author{
Cristina Tutuianu $^{1}$ - Vassil Traykov ${ }^{2}$ - Gábor Bencsik ${ }^{1} \cdot$ Gergely Klausz $^{1}$. \\ László Sághy $^{1} \cdot$ Robert Pap $^{1}$
}

Received: 29 June 2015 / Accepted: 14 October 2015 /Published online: 21 October 2015

(C) Springer Science+Business Media New York 2015

\begin{abstract}
Background Whether dissociated firing (DiFi) in isolated pulmonary veins (PVs) implies arrhythmogenicity of the particular PV and, therefore, a better outcome of PV isolation (PVI) for paroxysmal atrial fibrillation (PAF) is debated.

Methods Thirty-one patients undergoing their first PVI for PAF were studied. Isoproterenol was infused for induction, and the triggering PV was identified. During sustained PAF, sequential recordings were made with a decapolar circular mapping catheter from each PV. The dominant frequency (DF) was determined using fast Fourier transformation. Spontaneous DiFi was monitored for $30 \mathrm{~min}$ after PVI.

Results PAF was triggered by the PVs in all patients. Fourteen (45 \%) patients had DiFi after PVI in at least one PV. It was recorded most commonly from the left upper ( $84 \%$ ) and lower $(67 \%)$, less commonly from the right upper $(31 \%)$ PV. Out of the $23 \mathrm{PVs}$ with $\mathrm{DiFi}, 13$ (57\%) showed sporadic ectopic beats while $10(44 \%)$ had sustained ectopic rhythm or isolated tachycardia. There was no difference in size between PVs with or without DiFi (5.9 \pm 1.2 vs. $5.6 \pm 1.0 \mathrm{~cm}$ ostial perimeter, $p=$ $0.40)$. Triggering PVs more commonly showed any $\mathrm{DiFi}$, compared to nontriggering PVs (68 vs. $27 \%, p=0.003$ ) and more commonly had sustained DiFi (53 vs. $0 \%, p<0.001$ ). During PAF PVs with any DiFi showed faster maximal DF compared to PVs without DiFi (7.1 \pm 1.3 vs. $5.9 \pm 1.1 \mathrm{~Hz}, p=$ 0.001). Higher maximal DF was recorded in PVs with
\end{abstract}

Cristina Tutuianu

tutucris2008@yahoo.com

1 2nd Department of Medicine and Cardiology Center, University of Szeged, Semmelweis str. 6, 6725 Szeged, Hungary

2 Tokuda Hospital, Sofia, Bulgaria sustained versus sporadic DiFi versus PVs without DiFi (7.5 \pm 0.9 vs. $6.8 \pm 1.6$ vs. $5.9 \pm 1.1 \mathrm{~Hz}$, respectively, $p=0.002$ ). Patients with DiFi after PVI had a longer mean time to recurrent PAF compared to those without DiFi (52 vs. 32 months, $p=0.048$ ).

Conclusions Dissociated firing in isolated PVs is associated with their role in the initiation and maintenance of PAF.

Keywords Atrial fibrillation · Dissociated firing · Triggering pulmonary vein

\section{Introduction}

Pulmonary vein (PV) ectopy is recognized as a common trigger for the initiation of paroxysmal atrial fibrillation (PAF) [1]. Furthermore, a role for PVs in the maintenance of PAF has been suggested by frequency mapping [2]. Thereby, durable $\mathrm{PV}$ isolation (PVI) is a highly effective therapy when PV arrhythmogenicity is responsible for PAF. However, when other mechanisms are involved, the effectiveness of PVI may be limited [3].

After achieving PVI, dissociated firing (DiFi) from PVs is frequently observed [4]. The capability of PVs to generate an isolated ectopic rhythm may signify their arrhythmic potential and therefore predict a higher success [5]. On the other hand, the presence of DiFi may be related to better quality of PVI, farther away from PV ostia, also suggesting improved outcome [6]. Whether spontaneous electrical activity of isolated PVs is related to their arrhythmogenicity or only an epiphenomenon and whether observing DiFi after PVI has prognostic significance remain unclear. The purpose of this study was to investigate the association between DiFi and PV arrhythmogenicity by frequency mapping during PAF. 


\section{Methods}

\subsection{Patient characteristics}

Thirty-one consecutive patients undergoing their first PVI for drug-refractory, symptomatic PAF (53 \pm 9 years old, 17 men) were prospectively studied. All patients gave their written informed consent to participate in the study. Exclusion criteria were previous left atrial (LA) catheter ablation or open-heart surgery, persistent AF, severe valvular heart disease, or LA thrombus. Patients without inducible sustained PAF during the study (two patients) and those with PAF triggers originating from extra-PV sites (two patients) were also excluded.

\subsection{Electrophysiologic procedure}

Patients were on oral anticoagulation for at least 3 weeks before the ablation, and transesophageal echocardiography was performed to exclude any atrial thrombi before the procedure. All antiarrhythmic drugs were discontinued for at least five half-lives except amiodarone which was discontinued 1 month prior to the procedure. The study was performed as described previously [2]. Briefly, under conscious sedation (midazolam \pm fentanyl), following femoral venous access, two decapolar steerable catheters (interelectrode spacing 2-5-2 mm, Dynamic Deca; Bard Electrophysiology, Lowell, MA, USA) were positioned in the coronary sinus and the posterolateral right atrium. The left atrium (LA) and the PVs were mapped through double transseptal puncture. Intracardiac echocardiography (ICE) (AcuNav; Acuson Corp., Mountain View, CA, USA) was used for performing the transseptal puncture and to guide catheter positioning. A decapolar circular mapping catheter (CMC) (Inquiry Optima; St. Jude Medical, Irvine, CA, USA) and a 3.5-mm irrigated-tip mapping catheter (Navistar Thermocool; Biosense Webster, Diamond Bar, LA, USA) were advanced into the LA.

\subsection{Assessment of the triggering PVs and signal analysis}

The CMC was positioned at the left PV antrum overlying both left PVs, and the mapping catheter was positioned on the right PV carina. If the patient presented in sinus rhythm, PAF was induced by isoproterenol infusion, starting at $3 \mu \mathrm{g} / \mathrm{min}$ with incremental doses of $5 \mu \mathrm{g} / \mathrm{min}$ until PAF was induced, the maximum dose of $20 \mu \mathrm{g} / \mathrm{min}$ was reached, or the patient developed side effects. If the patient was in AF, first, we performed transthoracic electrical cardioversion before PAF induction. Ectopic activity triggering a PAF episode was identified, and the origin was determined based on the endocardial activation sequence and by comparison to paced activation sequences from PVs as well as observing LA-PV electrogram reversal as previously described [2]. Triggering activity was considered to originate from the right PVs when earliest activation and LA-PV electrogram reversal were recorded using the mapping catheter at the right PV carina. When earliest activation was recorded using the CMC, left upper or lower PV origin was determined based on the radiographic position of poles recording the earliest activity. After induction, isoproterenol administration was stopped and further recordings were made after a 5-min washout period. During sustained PAF, the CMC was used to record sequentially from each PV ostium. Signals were recorded for at least $30 \mathrm{~s}$ at a sampling rate of $997 \mathrm{~Hz}$ using a digital EP recording system (GE CardioLab; General Electric, Milwaukee, WI, USA) and were stored for offline analysis. Intracardiac recordings were analyzed, utilizing a custom-designed computer application prepared with the LabView software package (National Instruments, Austin, TX, USA). Signals were filtered between 30 and $500 \mathrm{~Hz}$, rectified, and low-pass filtered at $20 \mathrm{~Hz}$. A fast Fourier transformation (FFT) was performed on two consecutive 5-s episodes from each bipole of the CMC. The frequency spectrum in the $3-15 \mathrm{~Hz}$ range was obtained, and the peak with the highest power was determined as the dominant frequency (DF). Noisy and highly disorganized signals (organization index $<0.25$ ) were excluded from the analysis [2]. The DFs of consecutive 5-s episodes were averaged, and the maximum DF value of all bipoles was taken as the DF of that PV and used for analysis (Fig. 1).

\subsection{Pulmonary vein isolation}

Contiguous ablation lesions were delivered using the irrigated catheter (with a power setting of $25-35 \mathrm{~W}$ and $15 \mathrm{ml} / \mathrm{min}$ irrigation) to encircle ipsilateral PVs in pairs, with the end point of elimination or dissociation of PV potentials. Additional ablation inside the encircling lesion was delivered when needed to achieve this end point.

\subsection{Assessment of DiFi}

After electrical disconnection of all PVs, each PV was assessed periodically during a 30-min waiting period for the presence of DiFi. The CMC was positioned in one PV for $30 \mathrm{~s}$ and then moved to the next, repeating periodically during the waiting period. Rhythm in the PV was classified as follows: $1=$ absent (if there was no electrical activity at all), $2=$ sporadic DiFi (scarce and fortuitous occurrence of dissociated potentials without a regular rhythm), and $3=$ sustained DiFi (regular ectopic rhythm or isolated PV tachycardia) (Fig. 1b, c).

\subsection{Measurement of the PV ostial area}

The LA and PVs were segmented from high-resolution computed tomography (CT) volumes using the CARTO system (Biosense Webster, Baldwin Park, CA, USA), and cut planes were positioned to separate the PVs from the body of the LA. 


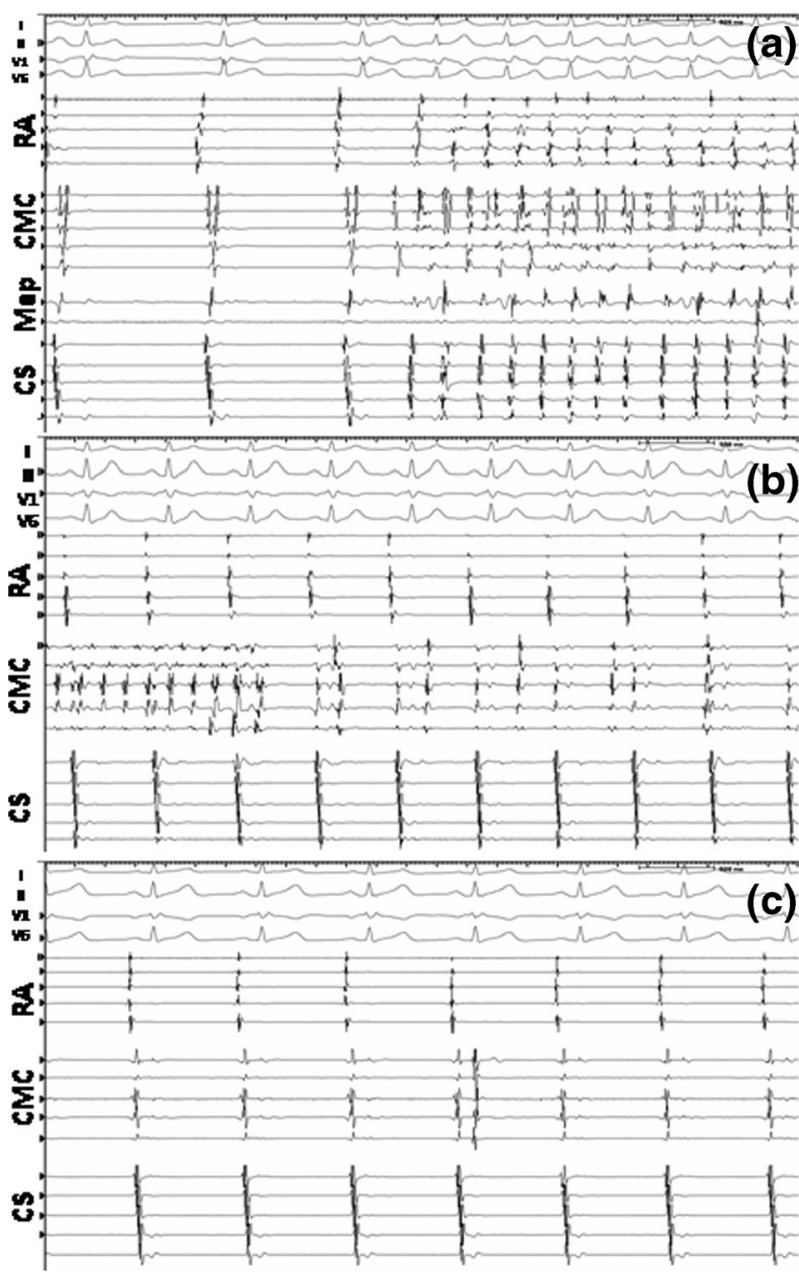

Fig. 1 Surface ECG leads I, II, $V_{1}$, and $V_{6}$, together with intracardiac recordings from the right atrium $(R A)$ and circular mapping catheter $(C M C)$ placed in the left pulmonary vein antrum (a), left superior pulmonary vein (LSPV, b), and left inferior pulmonary vein (LIPV, c) and from the proximal to distal coronary sinus $(C S)$. Atrial fibrillation is initiated by rapid discharge from the LSPV (a). Following isolation of the

Using area measurement tools of the CARTO system, we measured the cross-sectional area and the perimeter of the ostium of the PVs. We chose to determine the area of the ostium of PVs because it can be measured with greater precision than the mean diameter [7].

\subsection{Follow-up and redo procedures}

All patients had a follow-up visit at 3, 6, and 12 months. Seven-day Holter monitoring was performed when a patient had no symptoms at least 6 months after ablation to reveal asymptomatic recurrence or when patients reported symptoms suggestive of recurrence without documented arrhythmia. In case of recurrence, patients were offered a second procedure, during which reisolation of reconnected PVs was performed. No additional ablation of non-PV triggers or substrate outside the PV antra was undertaken. Since permanent isolation of

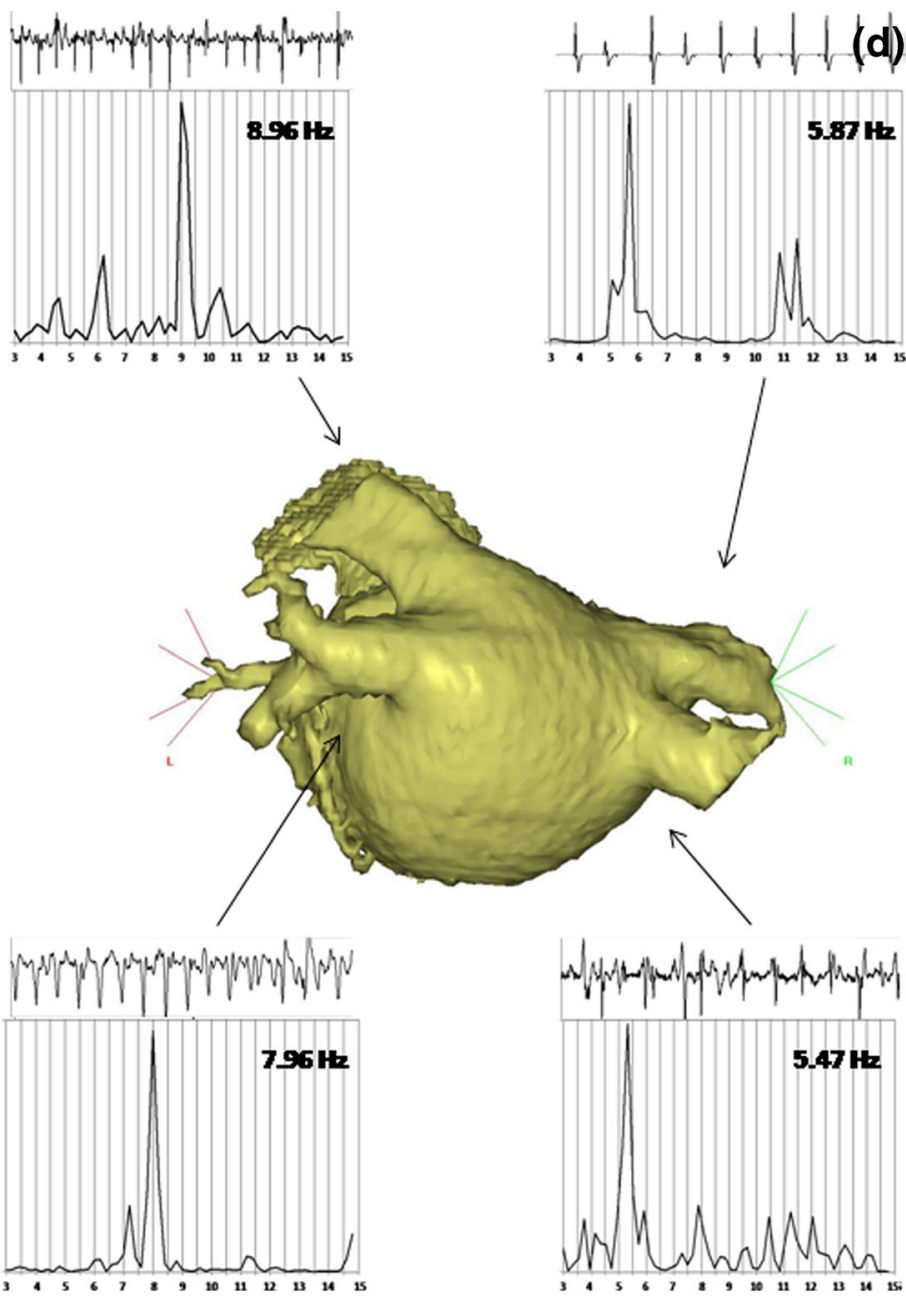

vein, sustained dissociated rhythm with intermittent burst activity was recorded (b). Sporadic dissociated activity was recorded in the LIPV after isolation (c). In the right pulmonary veins, there was no dissociated activity after isolation. Dominant frequency (DF) distribution during sustained atrial fibrillation in the same patient (d)

PVs often requires a second attempt, we evaluated the success of PVI after the last procedure and defined it as freedom from any sustained ( $>30 \mathrm{~s}$ ) atrial arrhythmia (symptomatic or asymptomatic), off antiarrhythmic drugs.

\subsection{Statistical analysis}

Continuous variables are reported as mean $\pm \mathrm{SD}$ and compared using one-way analysis of variance (ANOVA) and Student's $t$ test. Categorical variables are presented as percentage and compared using the chi-square test. Kaplan-Meier survival analysis was used to estimate the mean time to recurrent $\mathrm{AF}$ after the procedure. Survival curves were compared using the log-rank test. All statistical analyses were performed using the SPSS software version 16 (IBM Inc., NY, USA). A $p$ value $<0.05$ was considered statistically significant. 


\section{Results}

\subsection{Patient characteristics and incidence of DiFi after PVI}

Baseline characteristics of the patients from the study group are presented in Table 1. PAF triggers were found to originate from the left superior PV (LSVP) in 20 $(65 \%)$ patients, from the left inferior PV (LIPV) in 5 $(16 \%)$ patients, and from the right PVs in $6(19 \%)$ patients. Electrical isolation of PVs by circumferential ablation was achieved in all patients. Fourteen (45\%) patients had DiFi after PVI in at least one and 7 of them in more than one PV. It was recorded most commonly from the left upper (84\%) and lower (67\%) and less commonly from the right upper (31\%) PVs. Out of the $23 \mathrm{PVs}$ with DiFi, 13 (57\%) showed sporadic ectopic beats while 10 (44\%) had sustained ectopic rhythm or isolated tachycardia. No further ablation was performed to abolish this dissociated rhythm. There was no difference in size between PVs with or without DiFi $(5.9 \pm 1.2$ vs. $5.6 \pm 1.0 \mathrm{~cm}$ ostial perimeter, $p=0.40$, and $2.7 \pm 1.1$ vs. $2.4 \pm 0.9 \mathrm{~cm}^{2}$ ostial area, $p=0.55$ ).

\subsection{Association between PV triggers and DiFi}

Triggering PVs more commonly showed any DiFi, compared to nontriggering PVs ( 68 vs. $27 \%, p=0.003$ ) and more commonly had sustained DiFi (53 vs. $0 \%, p<0.001)$ (Fig. 2). Thus, the triggering vein was more likely to have dissociated ectopy after isolation.

\subsection{Association between DF and DiFi}

During sustained PAF, PVs with any DiFi showed faster maximal DF compared to PVs without DiFi (7.1 \pm 1.3 vs. $5.9 \pm$ $1.1 \mathrm{~Hz}, p=0.001)$. Higher maximal DF was recorded in PVs

Table 1 Clinical characteristics of patients with and without dissociated firing $(\mathrm{DiFi})$ after pulmonary vein isolation for paroxysmal atrial fibrillation (PAF)

\begin{tabular}{llll}
\hline Variable & No DiFi & DiFi & $p$ value \\
\hline Age (years) & $54 \pm 9.6$ & $52 \pm 8.5$ & 0.27 \\
Men (\%) & 41 & 71 & 0.19 \\
PAF duration (months) & $63.5 \pm 53.3$ & $58.9 \pm 67.4$ & 0.42 \\
Hypertension (\%) & 65 & 69 & 0.79 \\
Coronary disease (\%) & 6 & 8 & 0.84 \\
Diabetes (\%) & 12 & 8 & 0.71 \\
Left atrial diameter (mm) & $45.08 \pm 3.4$ & $45.45 \pm 6.9$ & 0.43 \\
LVEF (\%) & $61 \pm 6.28$ & $64.81 \pm 6.54$ & 0.08 \\
\hline
\end{tabular}

LVEF left ventricular ejection fraction

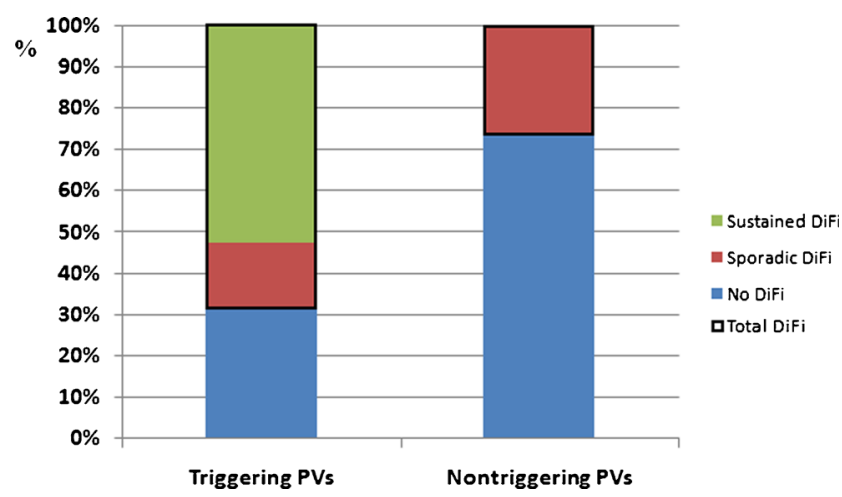

Fig. 2 Distribution of the different types of dissociated firing ( $D i F i)$ following isolation in pulmonary veins $(P V s)$ with atrial fibrillation triggers versus nontriggering PVs

with sustained versus sporadic DiFi versus PVs without DiFi (7.5 \pm 0.9 vs. $6.8 \pm 1.6$ vs. $5.9 \pm 1.1 \mathrm{~Hz}$, respectively, $p=0.002$ ) (Fig. 3). The maximal DF was higher in triggering PVs, compared to nontriggering ones $(8.02 \pm 1.64$ vs. $6.53 \pm 1.36$, $p<0.001)$.

\subsection{Clinical outcome}

During the ablation procedure and a mean of $31 \pm 18$ months of follow-up, no major complication occurred in any patient. Ten patients (32\%) underwent a redo PVI procedure, and all of them had reconnected PVs which were reisolated. There was no difference in the redo rate between groups (29 vs. $35 \%, p=0.690$, for patients with and without DiFi, respectively). Two patients (14\%) with DiFi and seven patients (41\%) without DiFi had a recurrence after the last procedure. One of the two patients with DiFi had asymptomatic recurrence and refused a second procedure. The difference in recurrence rate did not reach statistical significance $(p=0.101)$. However, patients with DiFi after PVI had a longer mean time to recurrent PAF after the last procedure compared to those without DiFi at the index procedure (52 vs. 32 months, $p=$ 0.048) (Fig. 4).

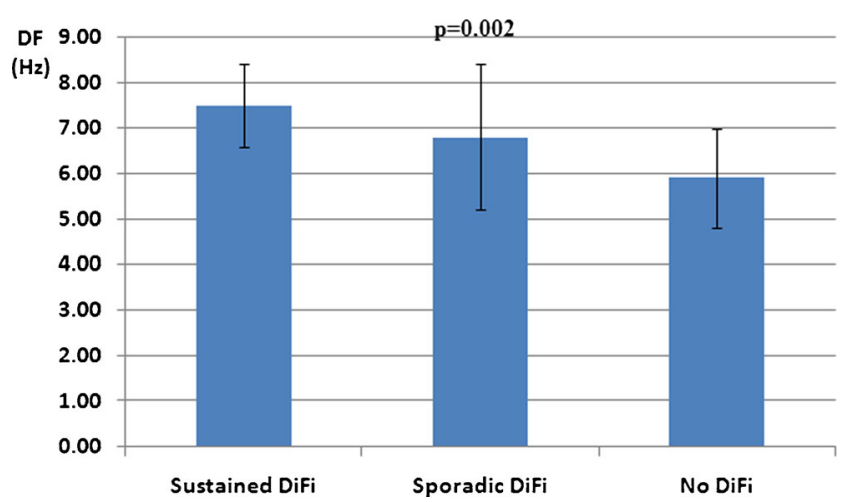

Fig. 3 Dominant frequency (DF) during atrial fibrillation in pulmonary veins showing different types of dissociated firing $(\mathrm{DiFi})$ after isolation 


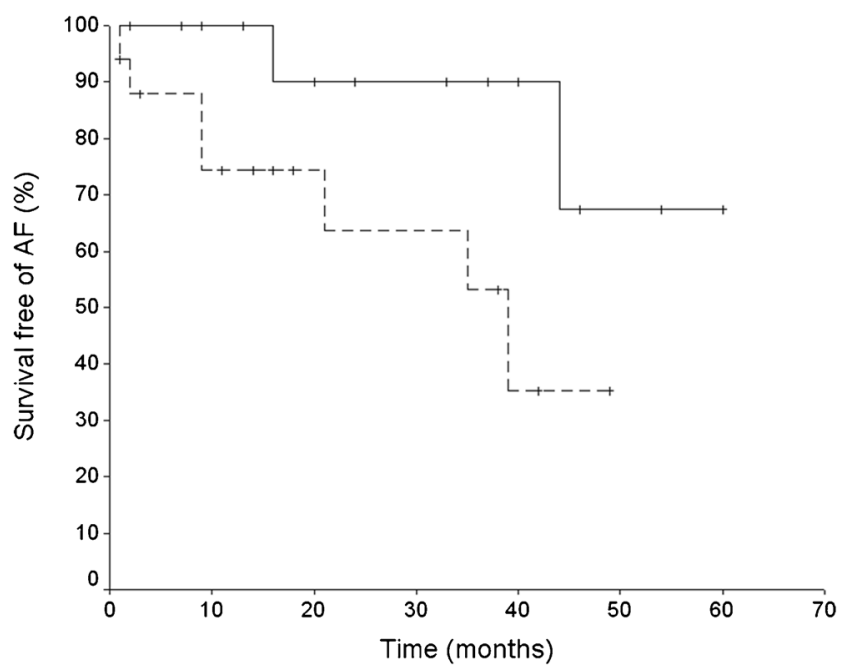

Fig. 4 Kaplan-Meier curve of the freedom from arrhythmia recurrence. Patients with dissociated firing (DiFi) after pulmonary vein isolation (continuous line) had a longer mean time to recurrence compared to those without DiFi (broken line)

\section{Discussion}

\subsection{Major findings}

The main finding of our study is that PVs with DiFi after isolation show higher activation rate during fibrillation and, therefore, are more likely to have a role in the perpetuation of atrial fibrillation as compared to PVs without DiFi. Furthermore, PVs showing DiFi more frequently initiate atrial fibrillation. Therefore, DiFi after PVI can be considered a hallmark of PV arrhythmogenicity pointing to the role of the particular PV both in the initiation and maintenance of PAF. When PAF is initiated and maintained by PV arrhythmogenicity rather than other mechanisms, PVI should have a better outcome.

\subsection{Mechanism of DiFi}

Spontaneous electrical activity of PVs after isolation presents as DiFi. It is either an escape rhythm overridden by the faster sinus node (SN) before isolation and unmasked by PVI or rapid ectopic triggering no longer capable of initiating PAF after isolation. Specialized cells resembling those of the SN have been described in the PVs and are thought to be responsible for spontaneous automaticity [8, 9] manifested as DiFi after PVI, which shows a pharmacological response similar to sinus rhythm [10]. However, PV electrical activity triggering PAF may have a mechanism that is different from the slow escape rhythm type of DiFi. Its mechanism is more compatible with triggered activity [11], being provoked by rapid pacing and the autonomic effects of adenosine [12], rather than being suppressed. This type of activity most commonly is eliminated by circumferential ablation around the PVs, possibly due to the effects of ablation on the autonomic nervous system [13]. Therefore, the association between DiFi and PV arrhythmogenicity deserves further investigation.

\subsection{Incidence of DiFi after PVI}

The incidence of DiFi after PVI shows a broad variation from 2.8 to $92 \%[10,14]$. This variation may be explained by differences in the definition of dissociated activity, study population, or ablation approach. Kabra et al. [14] reported an incidence of $92 \%$ of DiFi after PVI for PAF, and similar to our study, the DiFi was classified as isolated ectopic beats, ectopic regular rhythm, or PV fibrillation. In contrast, Buiatti et al. [15] in a recent study reported that $27 \%$ of their patients had at least one vein ( $12 \%$ of PVs) with DiFi, but the investigators took a unique approach to define the dissociated activity (slow intermittent potentials without a regular rhythm) and excluded most of what we have defined as DiFi. In our study, the incidence of DiFi was $45 \%$ of PAF patients presenting for PVI. Consistent with a previous study [16], we observed a higher proportion of DiFi originating from superior PVs compared to inferior veins. Also, PAF was triggered most commonly from upper PVs, similar to previous reports [17]. This may be related to thicker muscle sleeves [18]. Guerra et al. [19] linked areas of PV wall thickening to highfrequency potentials and the origin of ectopic beats. We could not find any significant correlation between the size of PVs and the presence of dissociated activity after isolation.

The ablation technique also has an impact on the occurrence of DiFi after PVI. Segmental, ostial isolation resulted in a DiFi rate of 5-33\% [4, 20], while wide-area encircling ablation resulted in up to $85-92 \%[14,21,22]$. This suggests that ablation closer to or inside the PV ostium (e.g., at the carina) can destroy some of the foci responsible for DiFi.

\subsection{Prognostic implications of DiFi}

Similar to the above mentioned reports defining the incidence and characteristics of DiFi, studies on the impact of dissociated activity on the outcome of PVI are also conflicting. Some have shown improved outcome of PVI in patients with DiFi $[5,6]$ and better success rate when the foci of DiFi were ablated inside the PVI lesions, rather than left untouched [23]. However, others have reported either no difference [15, $16,22]$ or even increased recurrence rate in the case of DiFi that is not ablated [23]. The explanation for an improved success rate in the aforementioned studies is either that DiFi is a marker of more proximal and better quality ablation lesions, providing evidence of exit block from the PVs [6] or that DiFi is a marker of PV arrhythmogenicity [5]. 


\subsection{Relation between DiFi and PV arrhythmogenicity}

Although, in two previous studies, the association between triggering PVs and DiFi was assessed [15, 22], a systematic approach to PAF induction was not employed and the proportion of patients with the triggering structure identified was low (10 and $44.5 \%$, respectively). Furthermore, the definition of triggering and DiFi was variable. One of these studies [15] suggested an association between PVs with PAF triggers and DiFi, while in the other, it was not significant [22]. We included only patients with the triggering PV identified (defined as the vein from which ectopic activity initiated PAF) and observed a significantly higher incidence of DiFi after isolation of a triggering PV, compared to nontriggering ones. This suggests that PVs with DiFi after PVI are more likely to have a role as initiators of PAF.

We previously described [2] that triggering PVs showed the fastest activity during sustained PAF, pointing to their role not only in the initiation but also in the perpetuation of the arrhythmia. In this study, we observed that PVs with DiFi showed faster maximal DF during PAF compared to PVs without DiFi, suggesting an association between DiFi and the maintenance of PAF. In addition, the correlation between DiFi and arrhythmogenicity of a PV both as initiator and perpetuator of PAF became more pronounced with more expressed (sustained vs. sporadic) DiFi. In line with the above and similar to studies showing DiFi to be a positive predictor of success [5, 6], we found that patients with DiFi after PVI had a longer mean time to recurrent PAF compared to those without DiFi.

These results confirm that observing DiFi from isolated PVs is related to the arrhythmogenicity of the PV. The presence of DiFi is associated with a better outcome of PVI not because the quality of PVI is higher but because it implies that PAF is more likely to have a PV-based mechanism for the particular patient. On the contrary, patients without DiFi after PVI are more likely to have non-PV mechanisms and more advanced atrial substrate involved in the arrhythmia. This is further supported by previous studies finding more structural heart disease [4, 24], hypertension [5], and non-paroxysmal atrial fibrillation [24] among cases without DiFi and a trend for a lower left ventricular ejection fraction in this study (Table 1).

\subsection{Limitations}

This study has several limitations. First, only patients in whom PAF could be induced and originated from the PVs were included; thereby, the real incidence of $\mathrm{DiFi}$, including those without inducible PAF and non-PV triggers, may be different. Second, the localization of PV triggers was done with approximation, because of the limited number of catheters available in the left atrium, due to ethical considerations. However, in our previous study, this method showed accurate detection of arrhythmogenic PVs [2]. Third, some patients received ablation at the PV carina which could have destroyed some of the foci responsible for DiFi. Fourth, the PVs were sequentially and intermittently checked for DiFi during the 30-min waiting period. It is possible for sporadic DiFi to be missed during the repositioning of the CMC. Fifth, the effect of the CMC on some observed DiFi cannot be completely ruled out. Sixth, pharmacological challenge was not employed to induce DiFi in silent veins after PVI. Although adenosine can rarely induce ectopic beats in isolated PVs, it mostly has a suppressant effect on $\mathrm{DiFi}[10,12]$. Isoproterenol is known to decrease the cycle length of DiFi [10] but never has been shown to induce DiFi in silent PVs. Since cycle length was not part of the definition of DiFi, the lack of isoproterenol testing after PVI was not expected to influence our results. Furthermore, our aim was to clarify the implications of spontaneous DiFi, a common finding after empirical PVI. Finally, the sample size was limited and this may have accounted for the lack of a statistically significant difference in success rates.

\subsection{Clinical implications}

The present study demonstrates an association between PV arrhythmogenicity and DiFi. PVs with DiFi are more likely to have a role as initiators and perpetuators of PAF and therefore deserve meticulous care in isolation and PVI verification (e.g., pace-capture or adenosine testing of the ablation lesion) to improve the long-term outcome of the procedure. On the contrary, in patients without DiFi after PVI, a more thorough search for non-PV mechanisms of arrhythmogenesis might be justified.

Conflict of interest The authors declare that they have no competing interests.

\section{References}

1. Haïssaguerre, M., Jaïs, P., Shah, D. C., Takahashi, A., Hocini, M., Quiniou, G., Garrigue, S., et al. (1998). Spontaneous initiation of atrial fibrillation by ectopic beats originating in the pulmonary veins. New England Journal of Medicine, 339(10), 659-666.

2. Traykov, V. B., Pap, R., Gingl, Z., Chadaide, S., Haqqani, H. M., Klausz, G., Gallardo, R., et al. (2013). Role of triggering pulmonary veins in the maintenance of sustained paroxysmal atrial fibrillation. Pacing and Clinical Electrophysiology, 36(7), 845-854.

3. Lin, W. S., Tai, C. T., Hsieh, M. H., Tsai, C. F., Lin, Y. K., Tsao, H. M., Huang, J. L., Yu, W. C., Yang, S. P., Ding, Y. A., Chang, M. S., \& Chen, S. A. (2003). Catheter ablation of paroxysmal atrial fibrillation initiated by non-pulmonary vein ectopy. Circulation, 107(25), 3176-3183.

4. Weerasooriya, R., Jaïs, P., Scavée, C., Macle, L., Shah, D. C., Arentz, T., Salerno, J. A., et al. (2003). Dissociated pulmonary vein 
arrhythmia: incidence and characteristics. Journal of Cardiovascular Electrophysiology, 14(11), 1173-1179.

5. Chen, H., Yang, B., Ju, W., Zhang, F., Hou, X., Chen, C., Zhai, L., et al. (2011). Long-term clinical implication of the occurrence of dissociated pulmonary vein activities after circumferential left atrial ablation in patients with paroxysmal atrial fibrillation. Circulation Journal, 75(1), 73-79.

6. Hussein, A. A., Ozaki, A., Martin, D. O., Bhargava, M., Baranowski, B., Dresing, T., Callahan, T., et al. (2013). Spontaneous dissociated firing from the pulmonary veins during ablation of paroxysmal atrial fibrillation: implications and impact on arrhythmia-free survival. Pacing and Clinical Electrophysiology, 36(8), 988-993.

7. Yuan, X. P., Bach, D., Skanes, A., \& Drangova, M. (2004). Assessment of intra- and interobserver variability of pulmonary vein measurements from CT angiography. Academic Radiology, 11(11), 1211-1218.

8. Chen, Y. J., Chen, S. A., Chang, M. S., \& Lin, C. I. (2000). Arrhythmogenic activity of cardiac muscle in pulmonary veins of the dog: implication for the genesis of atrial fibrillation. Cardiovascular Research, 48(2), 265-273.

9. Perez-Lugones, A., McMahon, J. T., Ratliff, N. B., Saliba, W. I., Schweikert, R. A., Marrouche, N. F., Saad, E. B., Navia, J. L., McCarthy, P. M., Tchou, P., Gillinov, A. M., \& Natale, A. (2003). Evidence of specialized conduction cells in human pulmonary veins of patients with atrial fibrillation. Journal of Cardiovascular Electrophysiology, 14, 803-809.

10. Marrouche, N., Wazni, O. M., Martin, D. O., Rossillo, A., Saliba, W., Erciyes, D., Schweikert, R., et al. (2005). Response to pharmacological challenge of dissociated pulmonary vein rhythm. Journal of Cardiovascular Electrophysiology, 16(2), 122-126.

11. Wit, A. L., \& Boyden, P. A. (2007). Triggered activity and atrial fibrillation. Heart Rhythm, 4(3 Suppl), S17-S23.

12. Cheung, J. W., Ip, J. E., Chung, J. H., Markowitz, S. M., Liu, C. F., Thomas, G., Lee, J. M., Lessner, S. J., \& Lerman, B. B. (2012). Differential effects of adenosine on pulmonary vein ectopy after pulmonary vein isolation: implications for arrhythmogenesis. Circulation. Arrhythmia and Electrophysiology, 5(4), 659-666.

13. Jiang, R. H., Jiang, C. Y., Sheng, X., Zhang, Z. W., Sun, Y. X., Liu, Q., $\mathrm{Fu}, \mathrm{G}$. S., et al. (2014). Marked suppression of pulmonary vein firing after circumferential pulmonary vein isolation in patients with paroxysmal atrial fibrillation: is pulmonary vein firing an epiphenomenon? Journal of Cardiovascular Electrophysiology, 25(2), $111-118$

14. Kabra, R., Heist, E. K., Barrett, C. D., Donaldson, D., Blendea, D., Beinart, R., Koruth, J., et al. (2010). Incidence and electrophysiologic properties of dissociated pulmonary vein activity following pulmonary vein isolation during catheter ablation of atrial fibrillation. Journal of Cardiovascular Electrophysiology, 21(12), 1338-1343.

15. Buiatti, A., Ammar, S., Reents, T., Semmler, V., Kathan, S., Hofmann, M., Bourier, F., et al. (2015). Dissociated pulmonary vein activity after pulmonary vein isolation for paroxysmal atrial fibrillation: a predictor for recurrence? Journal of Cardiovascular Electrophysiology, 26(1), 7-13.

16. Lee, G., Kalman, J. M., Vohra, J. K., Teh, A., Medi, C., Ling, L. H., \& Kistler, P. M. (2011). Dissociated pulmonary vein potentials following antral pulmonary vein isolation for atrial fibrillation: impact on long-term outcome. Heart, 97(7), 579-584.

17. Chen, S. A., Hsieh, M. H., Tai, C. T., Tsai, C. F., Prakash, V. S., Yu, W. C., Hsu, T. L., et al. (1999). Initiation of atrial fibrillation by ectopic beats originating from the pulmonary veins: electrophysiological characteristics, pharmacological responses, and effects of radiofrequency ablation. Circulation, 100(18), 1879-1886.

18. Nakagawa, H., Aoyama, H., Beckman, K. J., Po, S. S., Wu, R., Lockwood, D., Spector, P., et al. (2004). Relation between pulmonary vein firing and extent of left atrial-pulmonary vein connection in patients with atrial fibrillation. Circulation, 109(12), 1523-1529.

19. Guerra, P. G., Thibault, B., Dubuc, M., Talajic, M., Roy, D., Crépeau, J., Nattel, S., et al. (2003). Identification of atrial tissue in pulmonary veins using intravascular ultrasound. Journal of the American Society of Echocardiography, 16(9), 982-987.

20. Takahashi, Y., Iesaka, Y., Takahashi, A., Goya, M., Kobayashi, K., Fujiwara, H., \& Hiraoka, M. (2003). Reentrant tachycardia in pulmonary veins of patients with paroxysmal atrial fibrillation. Journal of Cardiovascular Electrophysiology, 14(9), 927-932.

21. Ouyang, F., Bänsch, D., Ernst, S., Schaumann, A., Hachiya, H., Chen, M., Chun, J., et al. (2004). Complete isolation of left atrium surrounding the pulmonary veins: new insights from the doubleLasso technique in paroxysmal atrial fibrillation. Circulation, 110(15), 2090-2096.

22. Miyazaki, S., Kuwahara, T., Kobori, A., Takahashi, Y., Takei, A., Sato, A., Isobe, M., et al. (2011). Prevalence, electrophysiological properties, and clinical implications of dissociated pulmonary vein activity following pulmonary vein antrum isolation. American Journal of Cardiology, 108(8), 1147-1154.

23. Doi, A., Satomi, K., Makimoto, H., Yokoyama, T., Yamada, Y., Okamura, H., Noda, T., et al. (2013). Efficacy of additional radiofrequency applications for spontaneous dissociated pulmonary vein activity after pulmonary vein isolation in patients with paroxysmal atrial fibrillation. Journal of Cardiovascular Electrophysiology, 24(8), 894-901.

24. Chen, S., Wu, H., Chen, G., Zhang, F., Meng, W., Yan, Y., Zhou, G., Qi, B., Xu, J., \& Liu, S. (2015). Clinical implications of and factors influencing dissociated pulmonary vein potentials. Journal of Cardiology, 66(2), 155-160. 